\title{
Epitope Spreading
}

National Cancer Institute

\section{Source}

National Cancer Institute. Epitope Spreading. NCI Thesaurus. Code C42597.

A normal feature of responses to infectious agents that allows recognition of multiple antigenic targets, Epitope Spreading involves non-cross-reactive epitopes, distinct from the inducing epitope, becoming major targets of an ongoing immune response. Epitope spreading (ES) involves an increased presentation of cryptic epitopes on one protein (intermolecular ES) or dominant epitopes on neighboring molecules (intramolecular ES). The result is an increase in antibody repertoire diversify, resulting in an enhanced overall immune response. Conversely, epitope spreading is the primary immunopathogenic event in autoimmune diseases. 